\title{
Visualization of the interplay between high-temperature superconductivity, the pseudogap and impurity resonances
}

KAMALESH CHATTERJEE ${ }^{1}$, M. C. BOYER ${ }^{1}$, W. D. WISE ${ }^{1}$, TAKESHI KONDO ${ }^{1,2 *}$, T. TAKEUCHI ${ }^{2,3}$, H. IKUTA2 AND E. W. HUDSON ${ }^{1 \dagger}$

\author{
${ }^{1}$ Department of Physics, Massachusetts Institute of Technology, Cambridge, Massachusetts 02139, USA \\ ${ }^{2}$ Department of Crystalline Materials Science, Nagoya University, Nagoya 464-8603, Japan \\ ${ }^{3}$ EcoTopia Science Institute, Nagoya University, Nagoya 464-8603, Japan \\ *Present address: Ames Laboratory and Department of Physics and Astronomy, lowa State University, Ames, lowa 50011, USA \\ †e-mail: ehudson@mit.edu
}

In conventional superconductors, the superconducting gap in the electronic excitation spectrum prevents scattering of low-energy electrons. In high-temperature superconductors (HTSs), an extra gap, the pseudogap ${ }^{1}$, develops well above the superconducting transition temperature $T_{\mathrm{C}}$. Here, we present a new avenue of investigating the pseudogap state, using scanning tunnelling microscopy (STM) of resonances generated by single-atom scatterers. Previous studies on the superconducting state of $\mathrm{HTSs}^{2}$ have led to a fairly consistent picture in which potential scatterers, such as $\mathrm{Zn}$, strongly suppress superconductivity in an atomic-scale region, while generating low-energy excitations with a spatial distribution - as imaged by $S_{T M}{ }^{3,4}$ —indicative of the $d$-wave nature of the superconducting gap. Surprisingly, we find that similar native impurity resonances coexist spatially with the superconducting gap at low temperatures and survive virtually unchanged on warming through $T_{\mathrm{C}}$. These findings demonstrate that properties of impurity resonances in HTSs are not determined by the nature of the superconducting state, as previously suggested, but instead provide new insights into the pseudogap state.

In $d$-wave superconductors, such as the high-temperature superconductors (HTSs), impurities act as pair breakers, giving rise to virtual bound states, or resonances, within the gap. For strong scatterers, these resonances lie close to the Fermi energy, and significantly modify bulk superconducting properties ${ }^{5,6}$. The local (atomic scale) effects of these resonances have been studied by several probes, such as nuclear magnetic resonance ${ }^{7-11}$ (NMR) and muon spin relaxation $(\mu \mathrm{SR})^{12}$. A variety of scanning tunnelling microscopy (STM) studies of impurity resonances in HTSs have been reported, including studies of native (unidentified) impurities ${ }^{13,14}$, intentionally doped $\mathrm{Zn}$ and $\mathrm{Ni}$ impurities $^{3,4}$ and intentionally placed surface impurities ${ }^{15}$. All of these STM studies demonstrated that impurity resonances are associated with an enhanced local density of states inside the gap, close to the Fermi energy. All of these studies were also carried out on $\mathrm{Bi}_{2} \mathrm{Sr}_{2} \mathrm{CaCu}_{2} \mathrm{O}_{8+x}(\mathrm{Bi}-2212)$ near $4 \mathrm{~K}$, significantly below $T_{\mathrm{C}}$.

Here, we report on temperature-dependent STM studies of native impurities in overdoped $\left(T_{\mathrm{C}}=15 \mathrm{~K}\right) \mathrm{Bi}_{2-\gamma} \mathrm{Pb}_{\gamma} \mathrm{Sr}_{2} \mathrm{CuO}_{6+x}$
(Bi-2201). In addition to enabling comparison to previous studies in Bi-2212, Bi-2201 has the benefit of having a relatively low $T_{\mathrm{C}}$, thus enabling us to study impurity resonances below and above $T_{\mathrm{C}}$ without the resonance being obscured by thermal broadening.

To carry out the temperature-dependent measurements discussed here we have constructed an ultrahigh-vacuum STM with the ability to track atomically resolved regions-here surrounding individual impurities - over a wide range of temperatures. We begin our study at low temperatures, using an experimental methodology similar to that used in previous STM impurity studies ${ }^{3,4}$. We search for impurity resonances by recording a spectral survey, in which differential conductance spectra (proportional to the local density of states) are recorded on a dense grid over a specified spatial region. In a spectral survey, impurity states are easily identified by their enhanced low-energy density of states.

After locating an impurity resonance, we zoom in and take a high spatial and energy resolution spectral survey to pinpoint the impurity's location, spectral shape and peak resonance energy. A high-resolution topograph and simultaneously acquired low-energy slice $G(\mathbf{r}, E=-2 \mathrm{meV})$ from such a survey are shown in Fig. 1. Spectral weight associated with the resonance appears predominantly at the locations of the centre and nextnearest-neighbour $\mathrm{Bi}$ atoms, which sit above $\mathrm{Cu}$ atoms in the superconducting $\mathrm{CuO}_{2}$ plane $\sim 5 \AA$ below (Fig. 1c).

After identifying the centre and shape of the impurity, we place the tip at specific locations and take energy-dependent differential conductance spectra. Figure 2 shows examples of such spectra taken at the resonance centre, as well as over nearest-neighbour and nextnearest-neighbour atoms. Spectra taken immediately outside the resonance show a typical gap structure, here of width $\Delta=11 \mathrm{meV}$. The edge of this gap may also be seen as small peaks in the central and next-nearest-neighbour spectra. The most obvious feature in these spectra, however, is a low-energy peak (centred at $\Omega=-2.75 \mathrm{meV}$ ). It is this peak's spatial dependence that is mapped in Fig. 1. In most theoretical treatments of impurity resonances, the energy of this main peak is determined by the strength of the potential scatterer ${ }^{2}$. Following Salkola et al. ${ }^{16}$, as has been done in previous analyses of STM results, and considering 

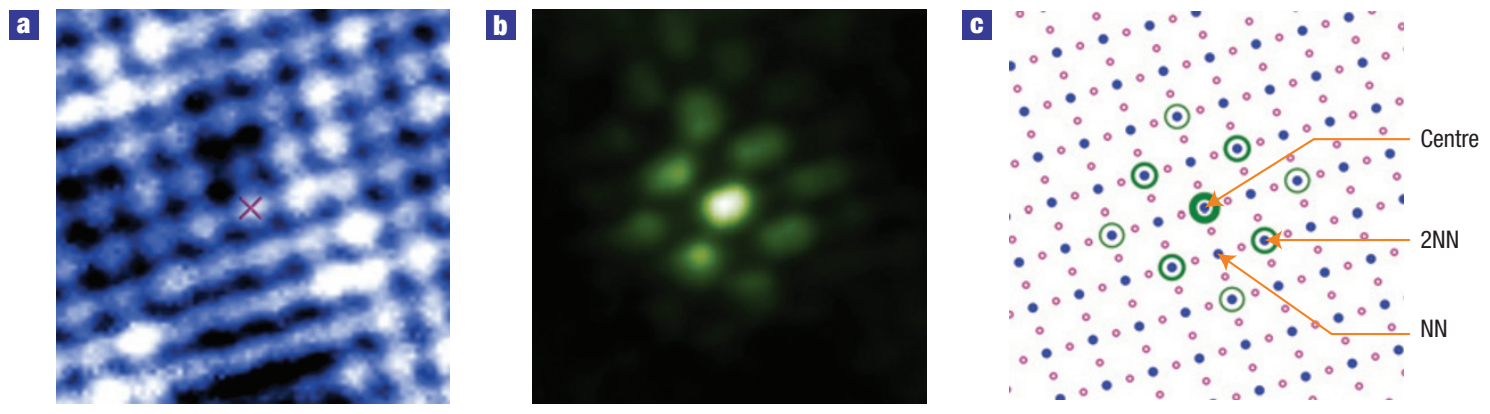

Figure 1 Topographic image and spectral map layer around the impurity resonance. a, $30 \AA$ square topographic image $\left(V_{\text {sample }}=-100 \mathrm{meV}, I=400 \mathrm{pA}, T=5.2 \mathrm{~K}\right)$ showing a BiO plane where the Bi- and the Pb-substituted atoms (brighter) are visible. Pb atoms have no observed associated spectroscopic signatures. b. Simultaneously acquired differential conductance map corresponding to $V_{\text {sample }}=-2 \mathrm{meV}$. The location of the bright centre of the impurity resonance is marked by the red ' $X$ ' on an otherwise indistinguishable $\mathrm{Bi}$ atom in the topography of $\mathrm{a}$. As $\mathrm{Cu}$ atoms in the $\mathrm{CuO}_{2}$ plane reside directly below the observed atoms, this is consistent with the native impurity being either a $\mathrm{Cu}$ vacancy or some element replacing $\mathrm{Cu}$ in the $\mathrm{CuO}_{2}$ plane. c, Schematic representation of the $\mathrm{CuO}_{2}$ layer underneath the $\mathrm{BiO}$ layer. $\mathrm{Blue}$ filled circles represent $\mathrm{Cu}$ atoms and pink open circles represent $\mathrm{O}$ atoms. The impurity centre is shown surrounded by the thickest green circle. The nearest-neighbour (NN) Cu atoms correspond to dark regions in the conductance map. Next-nearest-neighbour (2NN) Cu atoms have the second strongest signal in the conductance map.

that the resonance is in a local gap of width $\Delta=11 \mathrm{meV}$, we find that such a peak results from potential scattering with phase shift $\delta_{0}=0.4 \pi$ for the resonances reported here, compared with $0.48 \pi$ for $\mathrm{Zn}$ (ref. 4) and 'vacancies' (unidentified native scatterers) ${ }^{14}$ and $0.36 \pi$ for $\mathrm{Ni}$ (ref. 3 ). In the unitary (strongest scattering) limit, the phase shift would be $\pi / 2$. Thus, in this picture, these resonances are generated by strong, although not unitary, potential scattering.

Although the low-temperature behaviour of these resonances is very similar to those of $\mathrm{Zn}$ and vacancies in $\mathrm{Bi}-2212$, our temperature-dependent measurements yield surprising results: we find few significant changes in the impurity resonance as we warm through $T_{\mathrm{C}}$ into the pseudogap state. Figure 3 shows the temperature dependence of spectra from the resonance centre. Overlaying the data is the $5.2 \mathrm{~K}$ spectrum, thermally broadened to the appropriate temperatures (red lines). Thermal broadening is a purely mathematical procedure-convolution with the derivative of the Fermi function-that accounts for the decreased energy resolution of the STM at higher temperatures. Nearly all features of the higher-temperature data are well matched by the thermally broadened low-temperature data. This indicates that the resonance is not appreciably affected by temperature, and, in particular, not affected by the transition from the superconducting to the pseudogap state. Similarly, temperature-dependent conductance maps (Fig. 3b-d) taken at the same location as Fig. 1 show a lack of significant spatial change with warming.

From this temperature independence, we conclude that, contrary to previous assumptions, the spatial and energetic distribution of the impurity resonance is determined not by the superconducting gap but rather by the pseudogap. Although this interpretation differs from that of previous low-temperature STM results $^{3,4}$, it is consistent with recent experimental and theoretical work. Experimentally, a number of recent results suggest that the pseudogap not only exists above $T_{\mathrm{C}}$ but also coexists with the superconducting gap below $T_{C}$ (refs 17-20). Furthermore, NMR measurements have shown that the effects of a staggered paramagnetic polarization induced by $\mathrm{Zn}$ in $\mathrm{YBa}_{2} \mathrm{Cu}_{3} \mathrm{O}_{7}$ persist with little temperature dependence from above to below $T_{\mathrm{C}}$ (refs 9,21). Ellipsometry experiments have also demonstrated that impurities interact strongly with the pseudogap ${ }^{22}$, with $\mathrm{Zn}$ giving rise to a gradual and inhomogeneous pseudogap suppression, whereas Ni strongly enhances the pseudogap. Both the authors of that work and others ${ }^{23}$ have interpreted this as evidence of

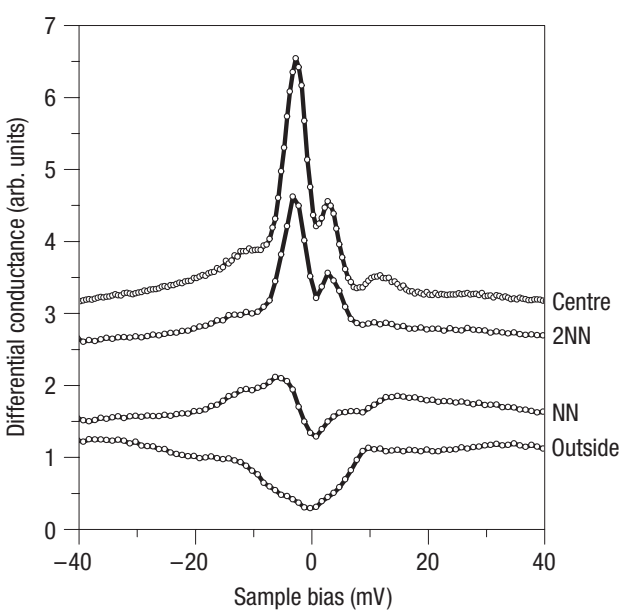

Figure 2 Tunnelling spectra taken at $5.2 \mathrm{~K}$ at different locations within the field of view shown in Fig. 1. The spectrum at the impurity centre (top curve) has a sharp resonance peak at $\Omega=-2.75 \mathrm{meV}$. The second peak at symmetric bias ( $\Omega^{\text {Sat }}=-\Omega=2.75 \mathrm{meV}$ ) is the 'satellite' peak and is probably due to the particle-hole symmetry of Bogoliubov quasiparticles. The spectra on next-nearest-neighbour $\mathrm{Cu}$ atoms are similar to those from the centre, with a reduction of the main peak amplitude. The spectra on nearest-neighbour Cu atoms indicate the presence of a gap instead of the impurity peaks found on adjacent atoms. A typical spectrum far from the impurity site is shown in the bottom plot. All spectra in this figure and in subsequent ones are taken with the same settings $\left(V_{\text {sample }}=-100 \mathrm{meV}, I=400 \mathrm{pA}, V_{\text {mod.r.m.s. }}=780 \mu \mathrm{eV}\right)$ and are vertically offset for clarity.

the importance of magnetic correlations in the pseudogap state. Tallon et al. have also stressed the importance of the pseudogap in interpreting the effects of impurities ${ }^{24,25}$.

Theoretically, several studies have discussed the possibility of impurity resonances in the pseudogap state. Kruis et al. ${ }^{26}$, for example, argue that neither phase coherence nor a particular gap shape is needed to generate a resonance peak near the Fermi energy. Instead, any depletion of the density of states at the Fermi energy is sufficient to produce resonance-like bound states near 

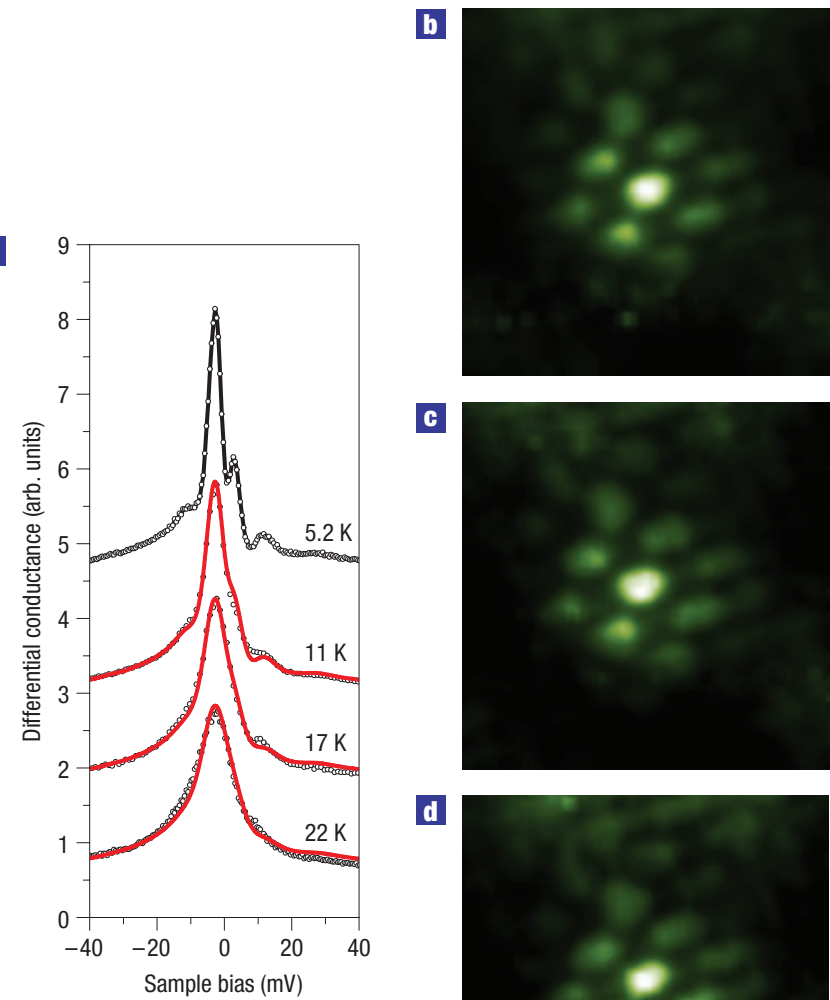

G

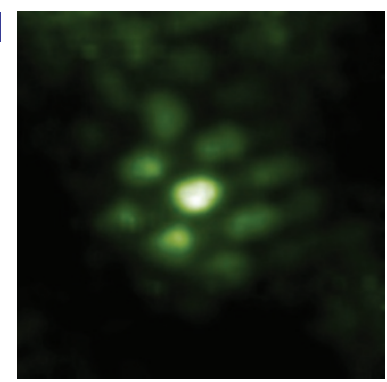

d

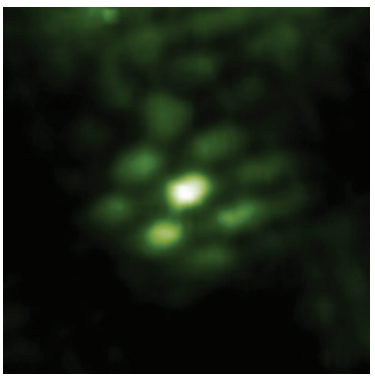

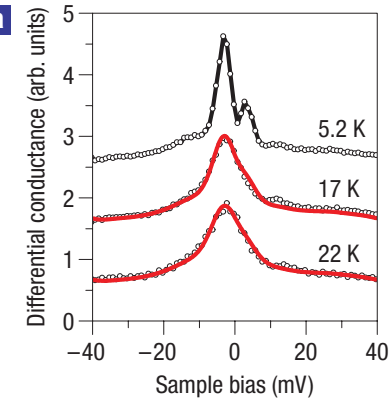
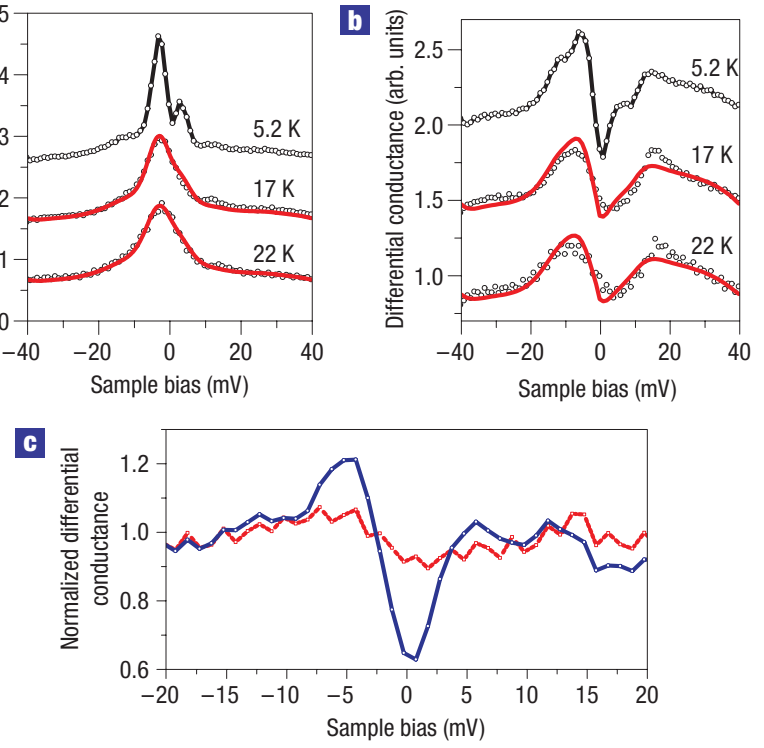

Figure 4 Temperature dependence of off-centre spectra through $T_{\mathrm{c}}$. a, Similar to spectra from the impurity centre (Fig. 3a), spectra from next-nearest-neighbour regions (black circles) show low-energy resonance peaks that are well matched by thermally broadened low-temperature data (red lines). b, In contrast, spectra from the nearest-neighbour Cu atoms (black circles) show instead a gap that is not well matched by the thermally broadened $5.2 \mathrm{~K}$ spectrum (red lines) above $T_{\mathrm{C}}=15 \mathrm{~K}$. c, The normalized $5.2 \mathrm{~K}$ spectrum of $\mathbf{b}\left(T_{\mathrm{N}}=22 \mathrm{~K}\right)$ (blue) reveals a small gap not found in the normalized $17 \mathrm{~K}$ spectrum (red), consistent with this gap being the superconducting gap.

Kruis et $a .^{26}$ instead focus on the 'satellite peak', a feature observable in the central and next-nearest-neighbour spectra of Fig. 2 opposite the main peak $\left(\Omega^{\mathrm{Sat}}=-\Omega=2.75 \mathrm{meV}\right)$. This peak has been interpreted as arising from particle-hole symmetry of Bogoliubov quasiparticles in a superconductor ${ }^{2,26}$, in which case its existence should be interpreted as a marker for the local presence of superconductivity. Thus, this peak should exist above $T_{\mathrm{C}}$ in the presence of phase fluctuations but not in the case of some other order. Unfortunately this is a difficult test, as thermal broadening quickly causes the satellite to merge with the central peak and we cannot from our data distinguish between the disappearance or simple broadening of the satellite. Further experimental studies on weaker impurities that generate higher-energy states, such as $\mathrm{Ni}$, should enable investigation of this proposal.

The clear presence of a satellite peak below $T_{\mathrm{C}}$ does however point towards the coexistence of superconductivity and the impurity resonance, as was previously observed near $\mathrm{Ni}$ atoms ${ }^{3}$. Another marker for superconductivity can be observed in the spectra (Figs 2, 4b) taken over the nearest-neighbour atoms (the intermediate dark regions in Fig. 1b). Here, a notable difference exists between spectra taken below $T_{\mathrm{C}}$ (at $5.2 \mathrm{~K}$ ) and those taken above (at $17 \mathrm{~K}$ and $22 \mathrm{~K}$ ) not captured by simple thermal broadening (Fig. 4b). To clarify the nature of this change, we divide the lower-temperature spectra by those taken at exactly the same location at a normalization temperature $T_{\mathrm{N}}$ above $T_{\mathrm{C}}$ (here $T_{\mathrm{N}}=22 \mathrm{~K}$ ). What remains (Fig. 4c, blue) is a small gap with half peak-to-peak separation $\Delta=5.5 \mathrm{meV}$, slightly smaller than the average $\Delta=6.7 \pm 1.6 \mathrm{meV}$ we recently reported from similarly normalized spectra taken away from impurities in indistinguishable samples ${ }^{19}$. In that work, we interpreted this gap, which is homogeneous and vanishes at $T_{\mathrm{C}}$, as the superconducting 
gap. Its existence here in the heart of the impurity resonance (on the nearest-neighbour atoms) is consistent with the observation of the satellite peak on the centre and next-nearest-neighbour atoms, and indicative of the coexistence of superconductivity and the resonance. That both the asymmetry of the main and satellite peaks and the reduction of the gap size $\Delta$ are consistent with a suppression of superconductivity is fascinating.

These results thus point towards an interesting interplay of the pseudogap and superconductivity in determining the nature of impurity resonances in HTSs. The energetic and spatial distributions of the main resonance seem to be set by the pseudogap, as they show no appreciable temperature dependence (except thermal broadening). On the other hand, the appearance and strength of the satellite peak and the size of the superconducting gap in the non-peaked (nearestneighbour) regions may be indicative of what remains of the superconducting state in the vicinity of the impurity. Continued research, particularly in samples intentionally doped with $\mathrm{Zn}$ or Ni, may further disentangle these effects and thus realize the potential of impurity studies to probe the nature of both superconductivity and the pseudogap in these complex materials.

\section{METHODS}

Our measurements are made using mechanically cut Pt-Ir (80\%-20\%) tips that are cleaned and sharpened through high-voltage $(800 \mathrm{~V})$ field emission on Au in ultrahigh vacuum at low temperatures. Before proceeding to measurements on a sample, we tunnel into Au to verify that the tip has a good work function and a flat density of states between at least $\pm 100 \mathrm{mV}$, an energy range larger than what is of interest for this study. Samples are cleaved in ultrahigh vacuum and at both high and low temperatures resulting in no noticeable topographic or spectroscopic differences with cleave temperature. Although we have observed similar impurity states using multiple samples and tips, for consistency of temperature dependent results, all scattering resonance data shown here are taken from a single impurity region.

\section{Received 1 October 2007; accepted 11 December 2007; published 20 January 2008.}

\section{References}

1. Timusk, T. \& Statt, B. The pseudogap in high-temperature superconductors: an experimental survey. Rep. Prog. Phys. 62, 61-122 (1999).

2. Balatsky, A. V., Vekhter, I. \& Zhu, J.-X. Impurity-induced states in conventional and unconventional superconductors. Rev. Mod. Phys. 78, 373-433 (2006).

3. Hudson, E. W. et al. Interplay of magnetism and high- $T_{\mathrm{c}}$ superconductivity at individual $\mathrm{Ni}$ impurity atoms in $\mathrm{Bi}_{2} \mathrm{Sr}_{2} \mathrm{CaCu}_{2} \mathrm{O}_{8+\delta}$. Nature 411, 920-924 (2001).

4. Pan, S. H. et al. Imaging the effects of individual zinc impurity atoms on superconductivity in $\mathrm{Bi}_{2} \mathrm{Sr}_{2} \mathrm{CaCu}_{2} \mathrm{O}_{8+\delta}$. Nature 403, 746-750 (2000).

5. Maeda, A., Yabe, T., Takebayashi, S., Hase, M. \& Uchinokura, K. Substitution of $3 \mathrm{~d}$ metals for $\mathrm{Cu}$ in $\mathrm{Bi}_{2}\left(\mathrm{Sr}_{0.6} \mathrm{Ca}_{0.4}\right)_{3} \mathrm{Cu}_{2} \mathrm{O}_{y}$. Phys. Rev. B 41, 4112-4117 (1990).
6. Kluge, T. et al. Clear distinction between the underdoped and overdoped regime in the $T_{c}$ suppression of Cu-site-substituted high- $T_{\mathrm{c}}$ cuprates. Phys. Rev. B 52, R727-R730 (1995).

7. Bobroff, J. et al. Persistence of $\mathrm{Li}$ induced Kondo moments in the superconducting state of cuprates. Phys. Rev. Lett. 86, 4116-4119 (2001).

8. MacFarlane, W. A. et al. Dynamics of the local moment induced by nonmagnetic defects in cuprates. Phys. Rev. Lett. 85, 1108-1111 (2000).

9. Ouazi, S. et al. Impurity-induced local magnetism and density of states in the superconducting state of $\mathrm{YBa}_{2} \mathrm{Cu}_{3} \mathrm{O}_{7}$. Phys. Rev. Lett. 96, 127005 (2006).

10. Tallon, J. L., Williams, G. V. M., Flower, N. E. \& Bernhard, C. Phase separation, pseudogap and impurity scattering in the HTS cuprates. Physica C 282-287, 236-239 (1997).

11. Williams, G. V. M., Tallon, J. L. \& Dupree, R. NMR study of magnetic and nonmagnetic impurities in $\mathrm{YBa}_{2} \mathrm{Cu}_{4} \mathrm{O}_{8}$. Phys. Rev. B 61, 4319-4325 (2000).

12. Nachumi, B. et al. Muon spin relaxation studies of $\mathrm{Zn}$-substitution effects in high- $T_{\mathrm{c}}$ cuprate superconductors. Phys. Rev. Lett. 77, 5421-5424 (1996).

13. Hudson, E. W., Pan, S. H., Gupta, A. K., Ng, K. W. \& Davis, J. C. Atomic-scale quasi-particle scattering resonances in $\mathrm{Bi}_{2} \mathrm{Sr}_{2} \mathrm{CaCu}_{2} \mathrm{O}_{8+\delta}$. Science 285, 88-91 (1999).

14. Hudson, E. W. et al. STM study of novel resonances in $\mathrm{Bi}_{2} \mathrm{Sr}_{2} \mathrm{CaCu}_{2} \mathrm{O}_{8+\delta}$. Physica B 329-333, 1365-1366 (2003).

15. Yazdani, A., Howald, C. M., Lutz, C. P., Kapitulnik, A. \& Eigler, D. M. Impurity-induced bound excitations on the surface of $\mathrm{Bi}_{2} \mathrm{Sr}_{2} \mathrm{CaCu}_{2} \mathrm{O}_{8}$. Phys. Rev. Lett. 83, 176-179 (1999).

16. Salkola, M. I., Balatsky, A. V. \& Scalapino, D. J. Theory of scanning tunneling microscopy probe of impurity states in a $d$-wave superconductor. Phys. Rev. Lett. 77, 1841-1844 (1996).

17. Le Tacon, M. et al. Two energy scales and two distinct quasiparticle dynamics in the superconducting state of underdoped cuprates. Nature Phys. 2, 537-543 (2006).

18. Tanaka, K. et al. Distinct Fermi-momentum-dependent energy gaps in deeply underdoped Bi2212. Science 314, 1910-1913 (2006).

19. Boyer, M. C. et al. Imaging the two gaps of the high-temperature superconductor $\mathrm{Bi}_{2} \mathrm{Sr}_{2} \mathrm{CuO}_{6+x}$. Nature Phys. 3, 802-806 (2007).

20. Kondo, T., Takeuchi, T., Kaminski, A., Tsuda, S. \& Shin, S. Evidence for two energy scales in the superconducting state of optimally doped $(\mathrm{Bi}, \mathrm{Pb})_{2}(\mathrm{Sr}, \mathrm{La})_{2} \mathrm{CuO}_{6+\delta}$. Phys. Rev. Lett. 98 , 267004 (2007).

21. Ouazi, S., Bobroff, J., Alloul, H. \& MacFarlane, W. A. Correlation length in cuprate superconductors deduced from impurity-induced magnetization. Phys. Rev. B 70, 104515 (2004).

22. Pimenov, A. V. et al. Nickel impurity-induced enhancement of the pseudogap of cuprate high- $T_{\mathrm{c}}$ superconductors. Phys. Rev. Lett. 94, 227003 (2005).

23. Dora, B., Maki, K., Virosztek, A. \& Vanyolos, A. Pseudogap enhancement due to magnetic impurities in $d$-density waves. Phys. Rev. B 75, 132504 (2007).

24. Tallon, J. L. Normal-state pseudogap in $\mathrm{Bi}_{2} \mathrm{Sr}_{2} \mathrm{CaCu}_{2} \mathrm{O}_{8}$ characterized by impurity scattering. Phys. Rev. B 58, R5956-R5959 (1998).

25. Tallon, J. L., Bernhard, C., Williams, G. V. M. \& Loram, J. W. Zn-induced $T_{\mathrm{c}}$ reduction in high- $T_{\mathrm{c}}$ superconductors: Scattering in the presence of a pseudogap. Phys. Rev. Lett. 79, 5294-5297 (1997)

26. Kruis, H. V., Martin, I. \& Balatsky, A. V. Impurity-induced resonant state in a pseudogap state of a high- $T_{c}$ superconductor. Phys. Rev. B 64, 054501 (2001).

27. Zhu, J.-X., Kim, W., Ting, C. S. \& Carbotte, J. P. Quasiparticle states around a nonmagnetic impurity in a $d$-density-wave state of high- $T_{c}$ cuprates. Phys. Rev. Lett. 87, 197001 (2001).

28. Morr, D. K. Resonant impurity states in the $d$-density-wave phase. Phys. Rev. Lett. 89, 106401 (2002).

29. Wang, Q.-H. Mechanism of pseudogap probed by a local impurity. Phys. Rev. Lett. 88, 057002 (2002).

30. Chakravarty, S., Laughlin, R. B., Morr, D. K. \& Nayak, C. Hidden order in the cuprates. Phys. Rev. B 63, 094503 (2001).

\section{Acknowledgements}

We thank Y. Wang for his help in the preparation of this manuscript and A. V. Balatsky, J. E. Hoffman, K. M. Lang, P. A. Lee, T. Senthil and X.-G. Wen for their helpful comments. This research was supported in part by a Cottrell Scholarship awarded by the Research Corporation, by the MRSEC program of the NSF under award DMR 02-13282, and also by NSF CAREER award DMR-034891. Correspondence and requests for materials should be addressed to E.W.H.

\section{Author contributions}

K.C., M.C.B. and W.D.W. shared equal responsibility for all aspects of this project from instrument construction to data collection and analysis. T.K. grew the samples and helped refine the STM. T.T. and H.I. contributed to sample growth. E.W.H. advised.

Reprints and permissions information is available online at http://npg.nature.com/reprintsandpermissions/ 